\title{
Influence of Surface Temperature Distribution on Reaction Rate of Solid Fuel Char Particles
}

\author{
Ewa Karchniwy ${ }^{1,2}$, Sławomir Sładek ${ }^{1}$, Adam Klimanek ${ }^{1}$, Andrzej Szlęk ${ }^{1}$, \\ Agnieszka Korus ${ }^{1}$, Wojciech Adamczyk ${ }^{1}$ \\ ${ }^{1}$ Department of Thermal Engineering, Silesian University of Technology \\ Konarskiego 22, 44-100 Gliwice, Poland \\ \{ewa.karchniwy; slawomir.sladek; adam.klimanek; andrzej.szlek; agnieszka.korus; wojciech.adamczyk\}@polsl.pl \\ ${ }^{2}$ Department of Energy and Process Engineering, Norwegian University of Science and Technology \\ Kolbjørn Hejes vei 1B, NO-7491 Trondheim, Norway
}

\begin{abstract}
In the paper the effect of surface temperature distribution on the reaction rate and conversion of char particles is investigated. The distribution of temperature at reacting char particles was observed experimentally and was attributed to the non-uniformity of chemical composition, porosity and shape of particles. Based on the measured temperature variation, the influence of the temperature non-uniformity on reaction rate at the particle surface was investigated analytically and numerically using a kinetic-diffusion model. It was found that the temperature non-uniformity translates into reaction rate variation with a temperature dependent standard deviation that has two extrema in the considered temperature range. However, the minimum lies in the region of high temperatures, where the bulk diffusion resistance limits the reaction rate. The effect was also investigated during combustion of char particles in a CFD model of a simple combustion chamber. It was shown that the non-uniform temperature has small but noticeable influence on mean reaction rate and particle conversion, leading to a larger distribution of conversion of solid fuel particles.
\end{abstract}

Keywords: reacting particle temperature, solid fuel combustion, char combustion, reaction rate, kinetic-diffusion model

\section{Introduction}

Coal is still, after oil, a second most important fuel in global primary energy supply. Its share comprises $38 \%$ in worldwide electricity generation [1] and $27 \%$ of World's primary energy consumption in 2019, however the contribution decreased to its lowest level since 2003 [2]. This is attributed to the continuous reduction in coal consumption in the OECD countries in favor of growth of natural gas consumption and the use of renewables. The demand for coal is, however, still increasing in many developing countries in Asia and Pacific regions [2]. Other solid fuels, whose share in the energy consumption is foreseen to be growing in the future, are wastes and biomass. It is therefore important, due to environmental, economic and energy efficiency reasons, to understand the processes involved in solid fuel conversion. Nowadays, much of the research and design of solid fuels combustion and gasification systems is carried out using mathematical modelling. The modelling tools should be reliable, and their predictions should reflect the investigated processes with satisfactory accuracy. In numerical modelling of combustion and gasification of pulverized solid fuels, it is usually assumed that the reacting particles have a single temperature, uniform throughout their volume [3]. As a result, surface reactions occur at the same rate over the entire particle surface. Consequently, also the flow of products of surface chemical reactions, known as Stefan flow, is also symmetric, and there is no net force acting on the particles due to this transport phenomenon. Similarly, in most of experimental studies, only a single mean particle surface temperature is measured, even for quite large particles. Such an approach was applied in the works [4]-[7], where valuable characteristics of $\mathrm{O}_{2} / \mathrm{N}_{2}$ and $\mathrm{O}_{2} / \mathrm{CO}_{2}$ combustion were provided, however the temperature distributions at particles' surfaces were not quantified. Küster et al. [8] applied Raman spectroscopy and thermal imaging to measure both, the temperature, and species distribution on milimeter-sized particles' surfaces in gasification conditions, and significant variation of surface temperature was observed in the measured data. The implications of this variation were not further studied. Recently Sładek et al. [9] measured surface temperatures of burning pulverized coal char (bituminous coal and lignite) particles $\left(100 \mu \mathrm{m}\right.$ ) in $\mathrm{O}_{2} / \mathrm{N}_{2}$ and $\mathrm{O}_{2} / \mathrm{CO}_{2}$ atmospheres using two-color pyrometry and observed considerable temperature non-uniformity at various combustion temperatures, and a weak dependence on fuel type. It was found that the temperature variation is increasing almost linearly with increasing mean particle temperature. The 
temperature non-uniformity was attributed to inhomogeneous composition and structure of the reacting char particles. The non-uniformity of Stefan flow induced by temperature distribution on particle surface was studied by Karchniwy et al. [10]. It was found that the acceleration of particle due to non-uniform Stefan flow is non-negligible and can influence the reacting particles' trajectories, however the effect is small.

In this study we focus on the influence of the particle temperature non-uniformity on reaction rate. First the influence was examined analytically, and then numerically, by studying the reaction rate and conversion of pulverized solid fuel particles in a simple combustion chamber.

\section{Analysis and Results}

The analysis is based on experimental results presented by Sładek et al. [9] and Karchniwy et al. [10], where it was found that the standard deviation of temperature at the particle surface $\sigma_{T}$ is a function of mean particle temperature $\bar{T}_{p}$. The function weakly depends on fuel type (bituminous and lignite coal chars were tested) and the resulting fit to the experimental data is [10]

$$
\sigma_{T_{p}}=0.187 \bar{T}_{p}-110.0
$$

where $\bar{T}_{p}$ should be provided in $\mathrm{K}$.

\subsection{Influence on the Reaction Rate}

In this section the influence of the temperature variation on the reaction rate during solid fuel particle combustion is presented. The analysis assumes that the solid fuel particle char is burning according to the kinetic-diffusion model, so that the reaction rate $\Theta$ is given by

$$
\Theta=\frac{\pi d_{p}^{2} p_{o x}}{m_{p 0}} \frac{1}{1 / R_{d}+1 / R_{k}}
$$

where $m_{p 0}$ is the initial particle mass, $d_{p}$ is the particle diameter, $p_{o x}$ is the partial pressure of oxidizer, $R_{k}$ is the chemical reaction rate and $R_{d}$ is the reaction rate due to diffusion defined as

$$
R_{d}=\frac{C}{d_{p}}\left(\left(T_{p}+T\right) / 2\right)^{3 / 4}
$$

where $C$ is a reaction-dependent constant $T_{p}$ is the particle temperature and $T$ is the temperature of the bulk gas. The kinetic rate is expressed as

$$
R_{k}=A \exp \left(-E / R T_{p}\right)
$$

where $A$ is the pre-exponential factor, $E$ is the activation energy, and $R$ is the universal gas constant. The kinetic rate $R_{k}$ is the apparent rate, thus the intrinsic reactivity and pore diffusion are already accounted for in parameters $A$ and $E$. In order to examine the influence of the particle temperature variation during combustion on the reaction rate the variance formula is applied, thus the standard deviation of the reaction rate can be expressed as

$$
\sigma_{\Theta}=\sqrt{\left(\frac{d \Theta}{d T_{p}} \sigma_{T_{p}}\right)^{2}}
$$

where the derivative of the reaction rate with respect to particle temperature is 


$$
\frac{d \Theta}{d T_{p}}=\frac{\pi d_{p}^{2} p_{o x}}{m_{p 0}} \frac{\frac{E \exp \left(E / R T_{p}\right)}{A R T_{p}^{2}}+\frac{3 d_{p}}{8 C\left(\left(T_{p}+T\right) / 2\right)^{7 / 4}}}{\left(\frac{\exp \left(E / R T_{p}\right)}{A}+\frac{d_{p}}{C\left(\left(T_{p}+T\right) / 2\right)^{3 / 4}}\right)^{2}}
$$

In Fig. 1 the standard deviation $\sigma_{\Theta}$ and the derivative $d \Theta / d T_{p}$ are shown in a function of temperature. Also, the resulting deviation in the reaction rate is presented. In the calculations the standard deviation of particle temperature, as given by Eq. (1), was used and the results correspond to combustion in air with $C=5.0 \mathrm{e}-12 \mathrm{sK}-3 / 4, A=0.002 \mathrm{~s} / \mathrm{m}, E=7.9 \mathrm{e} 7 \mathrm{~J} / \mathrm{kmol}$, $d_{p}=5 \mathrm{e}-4 \mathrm{~m}$, particle density $\rho_{p}=800 \mathrm{~kg} / \mathrm{m} 3$ and $T_{p}=T$.
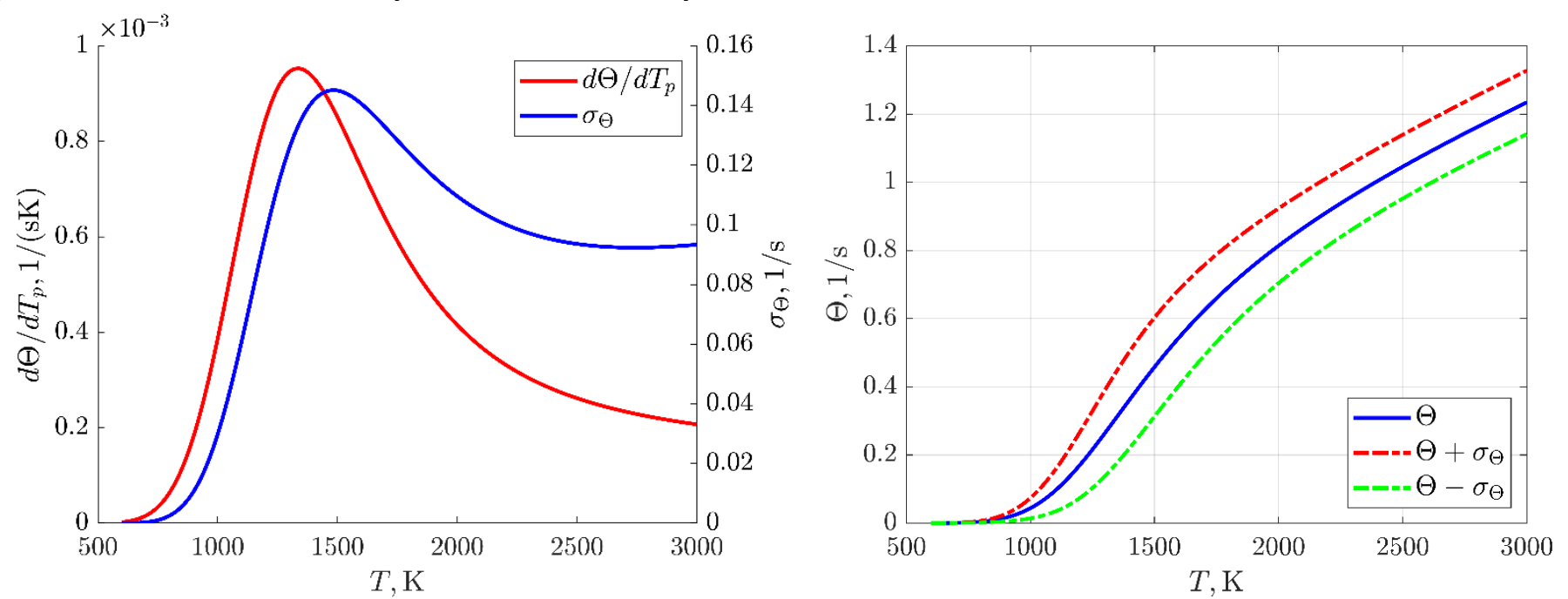

Fig. 1: The effect of temperature on derivative of reaction rate $d \Theta / d T_{p}$, standard deviations of the reaction rate $\sigma_{\Theta}$ and of particle temperature $\sigma_{T_{p}}$ (left) and on the reaction rate $\Theta$ (right).

As can be seen the standard deviation of the reaction rate is a nonmonotonic function and has two extrema in the selected temperature range. The second extremum is strictly linked with the increasing standard deviation of the particle temperature, however, it occurs at very high temperatures. The maximum obtained at around $1500 \mathrm{~K}$ indicates the highest influence of the variation of particle surface temperature variation at that temperature. A reduction of this influence at higher temperatures lies in the nature of the process, as at higher temperatures the diffusion of the reactant to the surface controls the overall reaction rate and thus the influence of temperature variation is limited. This effect can be also observed in Fig. 2, where the deviation from the mean is also shown. Due to this deviation in the reaction rate, the distribution of fuel conversion can be affected. This effect is examined in the next section.

\subsection{Influence on Fuel Conversion - CFD Model}

In the next step the influence of the temperature variation on reaction rate and fuel conversion in a combustion chamber was examined. For that purpose, a CFD model of a simple combustion chamber was developed using ANSYS Fluent code. Geometry of the domain is presented in Fig. 2. 


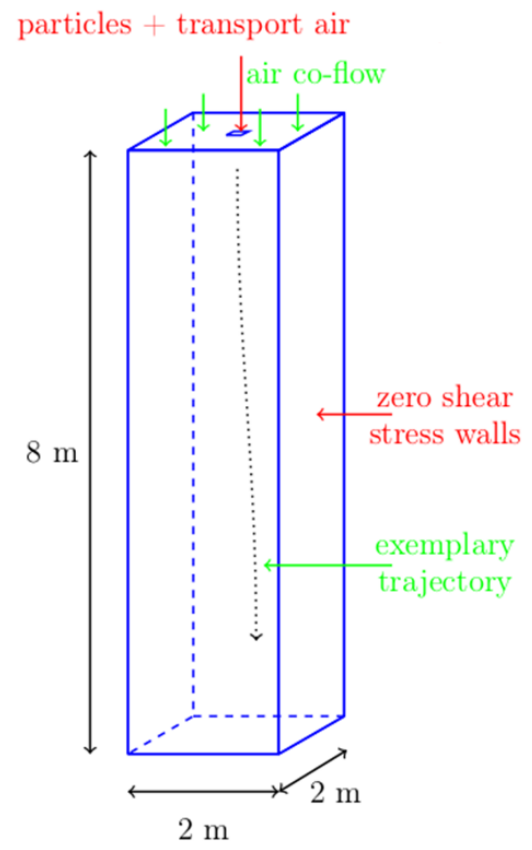

Fig. 2: Geometry of the model.

Simulations were performed for various co-flowing gas temperatures and oxygen concentrations in the oxidizer stream. In the CFD model energy conservation equation is computed for each particle. However, since the model assumes uniform composition and structure of the particles, the resulting temperature is treated as mean temperature $\bar{T}_{p}$. To incorporate the variation of temperature observed in the experiments, it was accounted for that the surface temperature of each particle can vary according to a normal distribution centered at $\bar{T}_{p}$ with the standard deviation given by Eq. (1). This modified temperature was then used in the rate expression (Eq. (2)). It was found that considerable dispersion of carbon conversion $C C$ occurred when the temperature non-uniformity at the particle surface was included in the reaction rate computations. In Fig. 3 sample results of such analyses are presented for combustion in air at temperature $T=1273 \mathrm{~K}$. In left panel of Fig. 3 the dispersion of carbon conversion is due to the spread of particles in the non-uniform flow, where $\mu_{C C}$ is the mean and $\sigma_{C C}$ is the standard deviation of the carbon conversion. In the right part of the figure, the distribution is increased due to surface temperature non-uniformity. It can be seen, that the standard deviation is much larger, which will also induce larger distribution of other flow variables in real combustion chambers.
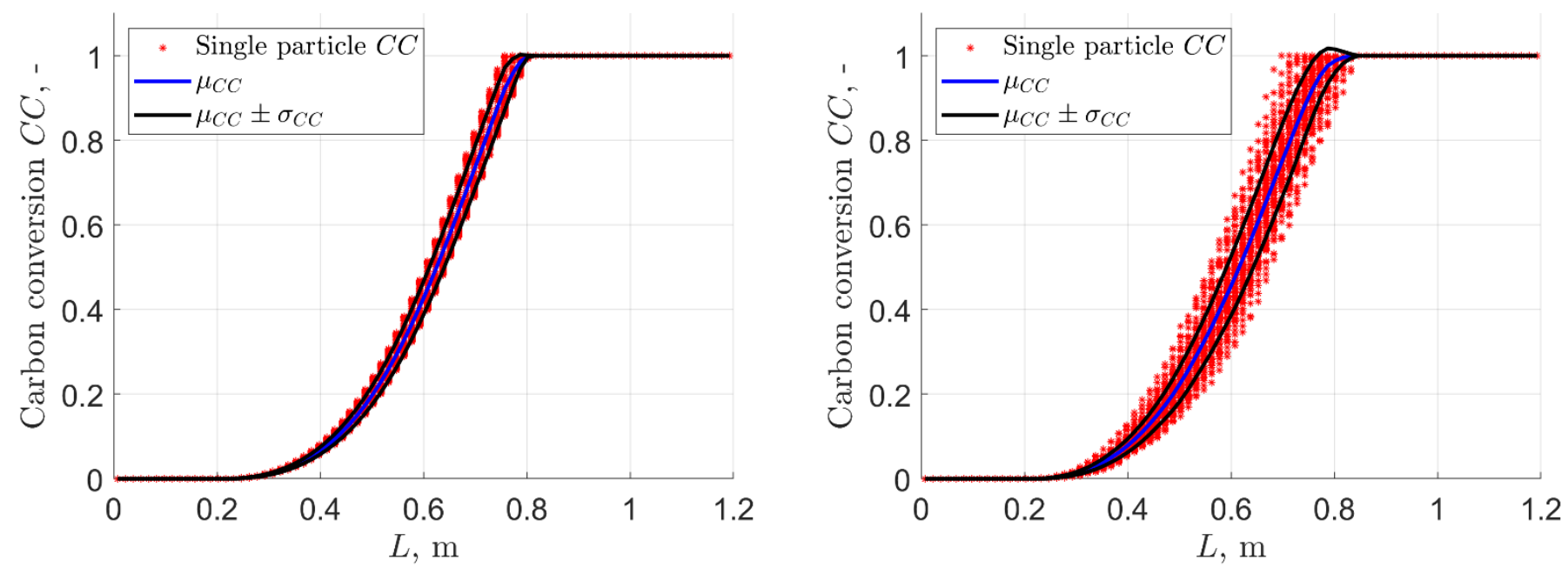
Fig. 3: Carbon conversion $C C$ for uniform (left) and non-uniform (right) temperature distribution at particle surface.

\section{Conclusion}

In the paper the effect of temperature distribution at reacting particle surfaces on the reaction rate and carbon conversion during solid fuel char combustion was investigated. The influence was examined by analysis of the variance in reaction rate induced by variance in temperature, using the variance formula and applying the kinetic-diffusion model for reaction rate. It was shown that the standard deviation of the reaction rate is a non-linear function of the mean particle surface temperature, despite the fact, that the deviation in the surface temperature is given by a linear expression. This translates into nonnegligible and temperature dependent distributions of the reaction rate. The influence of the surface temperature distribution on carbon conversion was then studied by CFD modelling of char particles combustion for a simple combustion chamber geometry. It was observed that a broader spatial dispersion of carbon conversion can be expected when the temperature nonuniformity is considered. The effect is not strong but noticeable. It is also expected that this broader dispersion will translate into redistribution of other flow variables in the combustion chamber.

\section{Acknowledgements}

The research has received funding from the Opus 13 Research Programme operated by the Polish National Science Center in the frame of Project Contract No UMO-2017/25/B/ST8/00957. This support is gratefully acknowledged.

\section{References}

[1] International Energy Agency, Coal 2018, IEA, Paris, [Online]. Available: https://www.iea.org/reports/coal-2018

[2] British Petroleum Company, Statistical Review of World Energy, 69 ${ }^{\text {th }}$ Edition, London: British Petroleum Co., 2020

[3] P. Edge, M. Gharebaghi, R. Irons, R. Porter, R.T.J. Porter, M. Pourkashanian, D. Smith, P. Stephenson, A. Williams, "Combustion modelling opportunities and challenges for oxy-coal carbon capture technology", Chemical Engineering Research and Design, 89, 9, 2011, pp. 1470-1493

[4] C.R. Shaddix, A. Molina, "Particle imaging of ignition and devolatilization of pulverized coal during oxy-fuel combustion", Proceedings of the Combustion Institute, 32, 2, 2009, pp. 2091-2098

[5] L. Zhang, E. Binner, Y. Qiao, Ch-Z. Li, "In situ diagnostics of Victorian brown coal combustion in O2/N2 and O2/CO2 mixtures in drop-tube furnace", Fuel, 89, 10, 2010, pp. 2703-2712

[6] R. Khatami, Ch. Stivers, K. Joshi, Y.A. Levendis, A.F. Sarofim, "Combustion behavior of single particles from three different coal ranks and from sugar cane bagasse in $\mathrm{O} 2 / \mathrm{N} 2$ and $\mathrm{O} 2 / \mathrm{CO} 2$ atmospheres", Combustion and Flame, 159, 3, 2012, pp. 1253-1271

[7] Y. Yuan, S. Li, Y. Xu, Q. Yao, "Experimental and theoretical analyses on ignition and surface temperature of dispersed coal particles in $\mathrm{O} 2 / \mathrm{N} 2$ and O2/CO2 ambients", Fuel, 201, 2017, pp. 93-98

[8] F. Küster, P. Nikrityuk, M. Junghanns, S. Nolte, A. Tünnermann, R. Ackermann, A. Richter, S. Guhl, B. Meyer, "Insitu investigation of single particle gasification in a defined gas flow applying TGA with optical measurements", Fuel, 194, 2017, pp. 544-556

[9] S. Sładek, A. Katelbach-Woźniak, W.P. Adamczyk, A. Klimanek, A. Korus, A. Szlęk, "Procedure for in-fly particle temperature detection under combustion conditions", Energy, 191, 2020, 116410

[10] E. Karchniwy, S. Sładek, A. Klimanek, A. Szlęk, W. Adamczyk, A. Korus, "The effect of surface temperature distribution on net force acting on reacting particles", in Proceedings of the 6th International Conference Contemporary Problems of Thermal Engineering. CPOTE 2020, Poland, 2020 